\title{
Risk of latent TB infection in individuals employed in the healthcare sector in Germany: a multicentre prevalence study
}

Anja Schablon*1,2, Melanie Harling1,2, Roland Diel³ and Albert Nienhaus²

\begin{abstract}
Background: Healthcare workers are still recognised as a high-risk group for latent TB infection (LTBI). Therefore, the screening of people employed in the healthcare sector for active and LTBI is fundamental to infection control programmes in German hospitals. It was the aim of the study to determine the prevalence and putative risk factors of LTBI.

Methods: We tested 2028 employees in the healthcare sector with the QuantiFERON-Gold In-tube (QFT-IT) test between December 2005 and May 2009, either in the course of contact tracing or in serial testing of TB high-risk groups following German OSH legislation.
\end{abstract}

Results: A positive IGRA was found in $9.9 \%$ of the healthcare workers (HCWs). Nurses and physicians showed similar prevalence rates (9.7\% to 9.6\%). Analysed by occupational group, the highest prevalence was found in administration staff and ancillary nursing staff (17.4\% and 16.7\%). None of the individuals in the trainee group showed a positive IGRA result. In the different workplaces the observed prevalence was 14.7\% in administration, $12.0 \%$ in geriatric care, $14.2 \%$ in technicians (radiology, laboratory and pathology), 6.5\% in admission ward staff and $8.3 \%$ in the staff of pulmonary/ infectious disease wards. Putative risk factors for LTBI were age ( $>55$ years: OR14.7, 95\% Cl 5.1-42.1), being foreign-born (OR 1.99, 95\% Cl 1.4-2.8), TB in the individual's own history (OR 4.96, 95\% Cl 1.99-12.3) and previous positive TST results (OR 3.5, 95\% Cl 2.4-4.98). We observed no statistically significant association with gender, BCG vaccination, workplace or profession.

Conclusion: The prevalence of LTBI in low-incidence countries depends on age. We found no positive IGRA results among trainees in the healthcare sector. Incidence studies are needed to assess the infection risk. Pre-employment screening might be helpful in this endeavour.

\section{Background}

The risk of latent tuberculosis infection (LTBI) and active tuberculosis (TB) as an occupational disease is well established and healthcare workers (HCWs) are still recognised as a high-risk group for LTBI [1-3]. Therefore, the screening of individuals employed in the healthcare sector for active TB and LTBI is fundamental to infection control programmes in hospitals, especially in low-incidence countries like Germany with a TB incidence of 6.1/ 100.000 in $2007[4,5]$. In accordance with the German

\footnotetext{
* Correspondence: anja.schablon@bgw-online.de

1 Institution for Statutory Accident Insurance and Prevention in the Health and Welfare Services, Department of Occupational Health Research, Pappelallee 35-37, 22089 Hamburg, Germany

Full list of author information is available at the end of the article
}

guidelines of occupational safety and health legislation $(\mathrm{OSH}) \mathrm{HCWs}$ are screened routinely depending on risk assessment. All HCWs working in pulmonary wards and labs (contact with contagious TB-material or TB cases) are screened every year. All other HCWs are routinely screened every third year. After exposure to a TB index case all close contacts were examined within the scope of contact investigation [6]. In accordance with the national guideline the Interferon-gamma release assay (IGRA) is used for the screening procedures. Early detection and treatment of LTBI are also recommended to prevent progression from latent TB infection to active TB [7].

So far, surveillance for LTBI in high-risk groups such as healthcare workers has been hampered by the non-speci- 
ficity of the tuberculin skin test (TST) in Bacillus Calmette-Guérin (BCG) -vaccinated individuals. However, there is no gold standard test for LTBI. For about a century the tuberculin skin test (TST) was the only available test for the detection of LTBI. But the TST has its limitations, including cross-reactivity to PPD (BCG-vaccination strain) and several non-tubercular mycobacteria (NTM) infections and the need for a second visit, which often results in missed follow-ups [8].

These limitations of the TST were overcome by the development of the new T-cell based in-vitro Interferon$\gamma$ release assays (IGRAs), which use $M$. tuberculosis-specific antigens $[3,9,10]$. These assays, commercially available in the form of QuantiFERON-TB and T-SPOT.TB, have been approved by the US Food and Drug Administration and endorsed in major national guidelines for use instead of or in addition to the TST for LTBI screening $[4,7,11,12]$. The third generation of the QuantiFERON test (QuantiFERON ${ }^{\circ}$ TB Gold In-Tube, QFT-IT) measures in-vitro IFN- $\gamma$ production by T-cells during in-vitro stimulation with peptides of the $M$. tuberculosis-specific antigens of the region of difference (RD-1) ESAT-6, CFP-10 and TB7.7. These antigens are not shared by any of the BCG vaccine strains nor by the more common species of NTM (e.g. M. avium) [13,14]. Available evidence reviewed elsewhere $[3,13,15]$ suggests that these IGRAs have higher specificity and at least an equal level of sensitivity as the TST and are unaffected by previous BCG vaccination and most NTM. Therefore this test reduce the risk of these tests overestimation due to cross-reactions with BCG vaccination or exposure to environmental mycobacteria [15].

So far, several systematic investigations of LTBI in HCWs using TST and IGRA have been published [1618], showing a high proportion of TST-positive/IGRAnegative results, which is most likely explained by BCG vaccination. A couple of studies had estimated the prevalence of LTBI in HCWs in low-incidence countries with the new in-vitro tests $[15,18-26]$. These prevalence rates are much lower than those assumed for German HCWs so far. The observed previous prevalence rate in German HCWs with the non-specific TST was 24\% [27].

It was the aim of our study to describe the prevalence in employees in the healthcare sector and the putative risk factors for LTBI.

\section{Methods}

\section{Study design and setting}

A cross-sectional study was conducted in 14 different kinds of hospitals in Germany. One of the clinics is a specialist lung disease clinic and the other clinics are general hospitals. Some of them also have special infection wards. The study population consisted of people employed in the healthcare sector tested between
December 2005 and May 2009, either in the course of a contact tracing or in serial testing of TB high-risk groups in accordance with German occupational safety and health legislation $(\mathrm{OSH})$ by occupational physicians. A total of 2028 employees in the healthcare sector were enrolled in the study. Of these, two smaller subgroups were already described in two studies by Nienhaus et al. $(\mathrm{n}=261)$ [26] and Schablon et al. $(\mathrm{n}=270)$ [24]. There were no exclusion criteria for study participants. The participants who had previous TB history were not excluded. The small sample size of $1.2 \%$ of participants with previous TB history did not affect the prevalence of LBTI in this study population:

The study protocol was approved by the ethics committee of the Hamburg Medical Council. All individuals gave their written informed consent prior to their inclusion in the study.

\section{Diagnostic methods}

For the IGRA, the QuantiFERON-TB Gold In-Tube test was used (Cellestis Limited, Carnegie, Australia). This whole-blood assay uses overlapping peptides corresponding to ESAT-6, CFP-10 and a portion of tuberculosis antigen TB7.7 (Rv2654). Stimulation of the antigenic mixture occurs within the tube used to collect the blood. Tubes were incubated at $37^{\circ} \mathrm{C}$ overnight before centrifugation, and INF- $\gamma$ release was measured by ELISA in accordance with the manufacturer's protocol. The QFT-IT was performed according to the manufacturer's instructions which consider a result to be positive if the IFN- $\gamma$ response of TB antigen minus Nil was $\geq 0.35 \mathrm{UI} / \mathrm{ml}$. The BCG vaccination status was confirmed by medical records or the presence of vaccination scars.

For the TST the intradermal Mendel-Mantoux test (two tuberculin units, $0.1 \mathrm{ml}$ purified derivate (PPD) RT 23, Staten Serum Institute, Copenhagen, Denmark) was used. Older TST results were conducted with the multipuncture test (GT 10, Behring Tuberculin). A TST with indurations $>5 \mathrm{~mm}$ was considered positive according to the German national guidelines [28].

\section{Questionnaire items}

Information on the following variables was collected using a standardised questionnaire: age, gender, reason for testing, occupational exposure to TB, time of occupation in the healthcare sector, family history of TB, place of birth, prior TST, job title, workplace, chest radiographic findings and BCG vaccination. BCG vaccination was assessed through the individual vaccination register or based on scars.

All participants with a positive QFT-IT were offered a clinical and radiological examination to rule out active TB. Treatment of LTBI with isoniazid for 9 months was proposed in accordance with the German recommendations. 


\section{Statistical analysis}

Data analysis was performed using SPSS, Version 14 (SPSS Inc., Chicago, Illinois). Chi-square tests were used for categorical data. Adjusted odds ratios (OR) and 95\% confidence intervals (CI) for QFT-IT, depending on different putative predictive variables, were calculated using conditional logistic regression. Model-building was performed backwards, using the chance criteria for variable selection [29]. There was a strong correlation between age and duration of employment. Therefore, we refrained from analysing both variables simultaneously.

\section{Results}

\section{Study population}

The study population comprises 2028 HCWs. 24 HCWs (1.2\%) were excluded from the analysis due to indeterminate QFT-IT results. The majority of the participating HCWs (77.2\%) were female and the mean age was 38.6 years $(\mathrm{SD} \pm 11.5)$. A history of BCG vaccination was recorded for $45.2 \%$ of the participants. $16.8 \%$ of the study population were born outside Germany or had a history of migration (Table 1). Most of the foreign-born participants came from former Soviet Union (NIS) states and Eastern Europe (12.5\%), 1.5\% came from Western European countries like Spain, France, Sweden, Portugal or Switzerland. 1.3\% were born in Asia, e.g. the Philippines, Thailand or India, and $1.0 \%$ of the participants immigrated from Africa or South America (no table). In the subgroup of participants from the former Soviet Union/ Eastern Europe, a positive IGRA was observed in $15.9 \%$ $(40 / 251)$, in the subgroup born in Western Europe the prevalence of a positive IGRA was $26.7 \%(8 / 30)$ and the highest prevalence of $37 \%$ was found in the subgroup born in Asia (10/27) (no Table). However, the differences were not statistically significant.

$54.2 \%$ of the total study population were nurses, $14.0 \%$ were physicians, and $5.5 \%$ were trainees or young professionals (physicians, medical staff, nurses, therapists). $3.5 \%$ were laboratory staff, a total of $4.6 \%$ were administration staff (including kitchen staff, secretaries, building services), 3.9\% were cleaning staff and $5.1 \%$ of the participants worked as social workers and auxiliaries (including medical secretaries, volunteers) (Table 1 ).

Within the scope of serial examinations of high-risk groups $68.7 \%(\mathrm{n}=1376)$ of HCWs were screened and 137 (10.0\%) of these participants were positive in the QFT-IT (Table 1). 628 employees (31.3\%) were screened in the course of contact tracing after exposure to a TB index case; in this group 9.7\% were QFT-IT positive (no table). We did not find any active TB cases.

A positive QFT-IT result was observed in 198 (9.9\%) participants and 480 (24.0\%) participants reported a positive TST (Table 1). Of the 480 participants with a positive previous TST, 94 (19.6\%) were confirmed by the IGRA
Previous TST results were mostly estimated with the old multipuncture test $(n=352)$. Out of 507 participants with no TST in their medical history, 48 persons (9.5\%) were positive by QFT-IT (Figure 1).

The prevalence of LTBI correlated with age. In the subgroup with participants under 25 years, LTBI prevalence was $1.6 \%$ and increased to $25.0 \%$ in the subgroup with participants over 55 years.

\section{Profession}

Regarding the prevalence of LTBI in different professions we found rates of $9.7 \%$ and $9.6 \%$ in nurses and physicians. None of the individuals in the trainee group showed a positive IGRA result. The highest prevalence was observed in the group of administration staff and ancillary nursing staff (including endoscopy and bronchoscopy staff and surgery staff) (17.4\% to $16.7 \%)$. In the known risk groups of technicians including radiology and laboratory staff, the observed prevalence varied from $3.6 \%$ to $11.4 \%$. The observed prevalence in the group of cleaning staff was $11.4 \%$. All IGRA-positive participants in this group have had a history of migration (no table).

\section{Workplace}

The observed prevalence in the admission ward was $6.5 \%$, compared to $8.3 \%$ in pulmonary/infectious disease wards. The highest prevalence was observed in administration staff $(14.7 \%)$, geriatric care staff $(12.0 \%)$ and technicians including radiology, laboratory and pathology staff (14.2\%). However, the differences were not statistically significant.

\section{Duration of employment in healthcare sector}

The prevalence of LTBI increased with the duration of employment, from $5.4 \%$ in the group with less than 5 years of employment up to $12.7 \%$ in the subgroup with more than 20 years in the healthcare sector. However, the correlation between duration of employment and age was high $\left(\mathrm{r}=0.665^{* * *}\right)$.

\section{Putative risk factors}

Putative risk factors for a positive IGRA result were age (>55 years: OR14.7, 95\% CI 5.1-42.1), being foreign-born (OR 1.99, 95\% CI 1.4-2.8) and TB in the individual's own history (OR 4.96, 95\% CI 1.99-12.3). Using the subgroup with a negative TST in their history as comparison group, the OR for those with no previous TST in their medical history was elevated $(1.7,95 \%$ CI 1.1-2.5). For those with previous positive TST results the OR was 3.5 (95\% CI 2.44.98). No statistically significant association was observed for gender, BCG vaccination, workplace and profession (Table 2).

Thus, so far no active TB has been diagnosed in any of the healthcare employees who had a positive QFT result during the study period. 
Table 1: Description of study population

\begin{tabular}{lcc}
\hline Variable & $\mathbf{N}=\mathbf{2 0 0 4}$ & $\%$ \\
\hline Age (years) & & \\
\hline$>25$ & 253 & 12.6 \\
\hline $25-35$ years & 481 & 24.0 \\
\hline $35-45$ years & $\mathbf{5 7 1}$ & $\mathbf{2 8 . 5}$ \\
\hline $45-55$ years & $\mathbf{5 0 7}$ & $\mathbf{2 5 . 3}$ \\
\hline$>55$ years & 192 & $\mathbf{9 . 6}$ \\
\hline Gender & & \\
\hline Female & $\mathbf{1 5 4 8}$ & $\mathbf{7 7 . 2}$ \\
\hline Male & $\mathbf{4 5 6}$ & \\
\hline
\end{tabular}

\section{Country of birth}

\begin{tabular}{lcc}
\hline Germany & 1667 & 83.2 \\
\hline Foreign-born & 337 & 16.8 \\
\hline
\end{tabular}

TB in own history

\begin{tabular}{lll}
\hline Yes & $\mathbf{2 4}$ & $\mathbf{1 . 2}$ \\
\hline No & $\mathbf{1 9 8 0}$ & $\mathbf{9 8 . 8}$
\end{tabular}

BCG vaccination

\begin{tabular}{lcc}
\hline Yes & 905 & 45.2 \\
\hline No & 1099 & 54.8 \\
\hline
\end{tabular}

Profession

\begin{tabular}{lcc}
\hline Nurse & 1087 & 54.2 \\
\hline Physician & 281 & 14.0 \\
\hline Trainee & 110 & 5.5 \\
\hline Therapist & 66 & 3.3 \\
\hline
\end{tabular}

\section{Discussion}

To the best of our knowledge, our study is the largest study to investigate the performance of the QFT-IT when screening healthcare staff in low-incidence countries like Germany. In this study we determined a low prevalence of positive QFT-IT results of 9.9\%. This prevalence was substantially lower compared to the findings assumed in the past with the TST (24\%)[27]. HCW with appositive TST were more often BCG vaccinated than those with a negative TST (66.2\% versus $39.2 \%)$. Therefore the discrepancy is most likely due to the BCG-vaccination.

In a further analysis of smaller subgroups of our study population, Nienhaus et al. investigated $261 \mathrm{HCWs}$ from different types of hospitals who are routinely screened for $\mathrm{TB}$ as stipulated by German OSH legislation using the QFT-IT and TST with a cut-off $>5 \mathrm{~mm}$. The prevalence of positive QFT-results was 9.6\% [26]. Schablon et al. investigated the prevalence of LTBI in a hospital for pulmonary diseases (in the northern part of Germany) which was also included in our study population. They found a prevalence of positive QFT-IT in $7.2 \%$ of the participants[24].

In line with this, Ringshausen and colleagues reported in another study a prevalence of LTBI of $9.1 \%$ among healthcare workers during a contact investigation in a hospital after close contact with a smear-negative index case [23]. In addition, Stebler et al. [22] investigated the prevalence of LTBI among employees at the hospital of Berne using the IGRA. A positive IGRA was carried out for 59/777 HCWs (7.6\%). A recent Australian study found comparable results for the frequency of positive QFT-IT (6.7\%) [18]. Harada et al. also observed the performance of the QFT-G for LTBI detection by testing $332 \mathrm{HCWs}$ in a Japanese general hospital and found a prevalence of positive QFT-G of 9.9\% [20].

In contrast to these findings, in the study among 95 HCWs working in departments of radiology, Barsegian et al. observed a prevalence of LTBI of $1 \%$ using the T-SPOT [21]. Also, Soborg and colleagues [19] investigated the prevalence at two departments of infectious diseases in Copenhagen using QFT- TB in 139 HCWs. 34 HCWs were office staff without routine patient contact and 105 HCWs had direct patient exposure. The observed prevalence in this study population was also 1\% compared to $34 \%$ with TST with a cut-off $>12 \mathrm{~mm}$. The positive QFTTB rate was much lower than the estimated prevalence in our study.

In a French study Tripodi and colleagues [30] compared the performance of the TST and IGRA in French HCWs when using a high cut-off for the TST ( $>10 \mathrm{~mm}) .18 .9 \%$ of the HCWs with recent contact to an acid-fast bacillus (AFB) -positive TB case were positive in the QFT. The rate of positive QFT is lower than the one observed in Portuguese HCWs (32.6\%) [31] but higher than the one 
Table 1: Description of study population (Continued)

\begin{tabular}{|c|c|c|}
\hline Administration & 92 & 4.6 \\
\hline Ancillary nursing staff & 30 & 1.5 \\
\hline Cleaning staff & 79 & 3.9 \\
\hline Social workers/auxiliary & 102 & 5.1 \\
\hline Radiology staff & 28 & 1.4 \\
\hline Laboratory staff & 70 & 3.5 \\
\hline Other & 59 & 2.9 \\
\hline \multicolumn{3}{|l|}{ Workplace } \\
\hline Admission ward & 138 & 6.9 \\
\hline Infection ward & 303 & 15.1 \\
\hline Geriatric care & 309 & 15.4 \\
\hline $\begin{array}{l}\text { Radiology/Laboratory/ } \\
\text { Pathology }\end{array}$ & 176 & 8.8 \\
\hline Administration & 75 & 3.7 \\
\hline Intensive care & 213 & 10.6 \\
\hline Clinic ward & 721 & 36.0 \\
\hline Other & 69 & 3.4 \\
\hline
\end{tabular}

History of TST

\begin{tabular}{lcc}
\hline Negative TST in history & $\mathbf{1 0 1 7}$ & $\mathbf{5 0 . 7}$ \\
\hline Positive TST in history & $\mathbf{4 8 0}$ & $\mathbf{2 4 . 0}$ \\
& & \\
\hline No TST & $\mathbf{5 0 7}$ & $\mathbf{2 5 . 3}$ \\
\hline
\end{tabular}

(10\%) observed in German HCWs [23,24,26], which corresponded with a higher TB incidence in the Portuguese general population.

In our study none of the individuals in the trainee group showed a positive IGRA result. This finding suggests that LTBI risk is very low at the beginning of the training period in the various occupations (nurses, medical students, physical therapists). Similar to our findings,
Chee et al. examined 270 medical students in Singapore in their final year of study. A positive T-Spot was found in $4.3 \%$ of the students. But this study was carried out in a country with intermediate tuberculosis incidence and it determined the prevalence at the end of training [32]. Therefore, the prevalence is somewhat higher than in Germany.

Another aim of our study was to determine putative risk factors for LTBI. We found that LTBI was associated with age ( $<25$ years $1.6 \%,>55$ years $25 \%)$, foreign birth and previous positive TST or no previous TST. The higher prevalence in older HCW/s was also observed in other studies $[20,26,33,34]$ and might be due to a cohort effect or the longer time at risk. These findings suggest that the IGRA describes the age dependency of LTBI prevalence in the German population very well. The association between foreign birth and LTBI was also observed on earlier analysis of a smaller subgroup of our study population published by Nienhaus et al. [26], but due to the smaller sample size the risk was not statistically significant.

We found no association between a positive QFT-IT and different kinds of occupations and workplaces. Rotation of the staff is an explanation for the lack of any association. We do not know how long the individuals were employed in a specific work area. In the course of their career, physicians and nursing staff work in various wards and hospitals; this makes it hard to attribute a positive test result to a specific work area. Furthermore, our cross-sectional design might dilute the expected association. We found no explanation for the high rate of positive QFT-IT results in the subgroup of the administration staff. We examined potential risk factors, e.g. age, migration, reason for testing (contact investigation), but no association for these putative risk factors was found. It should also be born in mind that our data are derived from routine screening of exposed HCWs. Therefore, even the seemingly unexposed group "administration" is not really unexposed.

Torres et al. investigated Portuguese HCWs with the TST and QFT-IT, and obtained findings similar to ours. Surprisingly, neither risk assessment (workplace) nor profession was associated with a positive TST or IGRA [31].

In contrast to our results, three biological studies showed a job-related exposure to TB for employees in the healthcare sector. Firstly, a molecular biological fingerprint study in Germany observed that the risk of active TB for HCWs is not higher compared to the general population. However, when the disease occurred, TB infection is most probably due to occupational exposure. In 8/ 10 HCWs who developed TB the infection was a consequence of occupational exposure. Secondly, Ong et al. found genotyping or epidemiological evidence of job- 


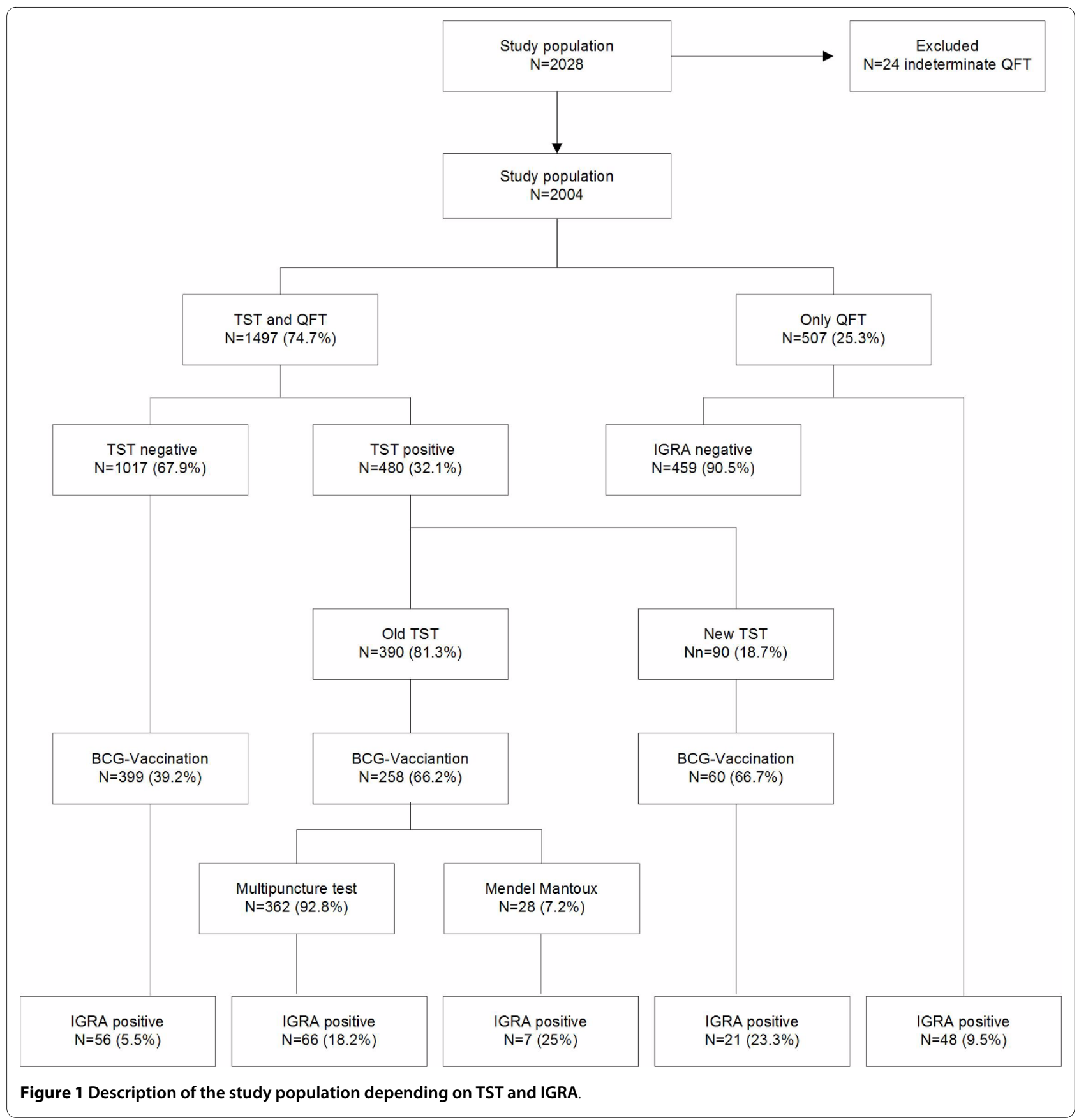

related transmission in at least $32 \%$ of HCWs [35]. And thirdly, de Vries and colleagues determined which TB cases among HCWs were caused by infections acquired at the workplace. Of a total of $101 \mathrm{~TB}$ cases, the infection pathways of 67 cases could be established; $42 \%$ were due to infection at work [36]. The majority of work-related active TB cases occurred when the infection risk was not suspected and preventive measures were not taken.

In a recent review occupational risk factors were linked to working in internal or respiratory medicine wards, duration of employment in the healthcare sector and $\mathrm{TB}$ exposure [3]. So far, the occupational risk of LTBI of different occupations has been investigated in several conventional studies. Most of these studies showed a significant increase of $>2$ in the relative risk for nurses. Several studies of varying quality examined the infection risk for physicians. Because of the inadequate data it is difficult to evaluate the LTBI risk in different medical specialties; the results do not indicate an increased infection risk for physicians. Only pathologists bear an increased LTBI risk [1]. 
Table 2: Frequency and adjusted odds ratios (OR) and $95 \%$ confidence intervals $(95 \% \mathrm{CI})$ for covariates associated with QFT-IT results

\begin{tabular}{|c|c|c|c|c|}
\hline Covariates & $\begin{array}{l}\text { QFT-IT } \\
\text { Negative n/\% }\end{array}$ & $\begin{array}{l}\text { QFT-IT } \\
\text { Positive n/\% }\end{array}$ & Odds Ratio & $95 \% \mathrm{Cl}$ \\
\hline \multicolumn{5}{|l|}{ Age ** } \\
\hline$>25$ years & $249(98.4)$ & $4(1.6)$ & 1 & \\
\hline $25-35$ years & $449(93.3)$ & $32(6.7)$ & 3.8 & $1.3-11.2$ \\
\hline $35-45$ years & $524(91.8)$ & $47(8.2)$ & 4.4 & $1.6-12.5$ \\
\hline $45-55$ years & $440(86.8)$ & $67(13.2)$ & 7.6 & $2.7-21.4$ \\
\hline$>55$ years & $144(75.0)$ & $48(25.0)$ & 15.1 & $5.3-43.4$ \\
\hline \multicolumn{5}{|l|}{ Gender** } \\
\hline Female & $1392(89.9)$ & $156(10.1)$ & 1 & \\
\hline Male & $414(90.8)$ & $42(9.2)$ & 1.2 & $0.8-1.7$ \\
\hline \multicolumn{5}{|l|}{ Country of birth ${ }^{* *}$} \\
\hline Germany & $1529(91.7)$ & $138(8.3)$ & 1 & \\
\hline Foreign-born & $277(82.2)$ & $60(17.8)$ & 2.0 & $1.4-2.8$ \\
\hline \multicolumn{5}{|l|}{ TB in own history** } \\
\hline No & $1792(90.5)$ & $188(9.5)$ & 1 & \\
\hline Yes & $14(58.3)$ & $10(41.7)$ & 4.9 & $1.97-12.2$ \\
\hline \multicolumn{5}{|l|}{ BCG vaccination } \\
\hline No & $995(90.5)$ & $104(9.5)$ & 1 & \\
\hline Yes & $811(89.6)$ & $94(10.4)$ & 0.9 & $0.7-1.3$ \\
\hline \multicolumn{5}{|l|}{ Profession } \\
\hline Physicians & $254(90.4)$ & $27(9.6)$ & 1 & \\
\hline Nurses & $982(90.3)$ & $105(9.7)$ & 0.94 & $0.6-1.6$ \\
\hline Administration staff & $146(85.4)$ & $25(14.6)$ & 1.1. & $0.5-2.2$ \\
\hline
\end{tabular}


Table 2: Frequency and adjusted odds ratios (OR) and $95 \%$ confidence intervals $(95 \% \mathrm{CI})$ for covariates associated with QFT-IT results (Continued)

\begin{tabular}{|c|c|c|c|c|}
\hline Technicians and special ward staff & $175(90.2)$ & $19(9.8)$ & 0.7 & $0.3-1.5-$ \\
\hline Unknown & $51(86.4)$ & $8(9.9)$ & 1.3 & $0.5-3.3$ \\
\hline Social workers/auxiliaries & $198(93.4)$ & $14(6.6)$ & 0.8 & $0.4-1.8$ \\
\hline \multicolumn{5}{|l|}{ Workplace } \\
\hline Other clinic ward & $653(90.6)$ & $68(9.4)$ & 1 & \\
\hline Unknown & $64(92.8)$ & $5(7.2)$ & 0.9 & $0.3-2.4$ \\
\hline Admission ward & $129(93.5)$ & $9(6.5)$ & 0.7 & $0.2-2.3$ \\
\hline Infection ward & $278(91.7)$ & $25(8.3)$ & 1.3 & $0.4-3.8$ \\
\hline Geriatric care & $272(88.0)$ & $37(12.0)$ & 1.7 & $0.6-4.9$ \\
\hline Technicians & $151(85.8)$ & $25(14.2)$ & 2 & $0.6-6.4$ \\
\hline Administration & $64(85.3)$ & $11(14.7)$ & 1.7 & $0.5-5.7$ \\
\hline Intensive care/surgery room & $195(91.5)$ & $18(8.5)$ & 1.1 & $0.9-3.3$ \\
\hline \multicolumn{5}{|l|}{ Previous TST in medical history** } \\
\hline Negative TST & $961(94.5)$ & $56(5.5)$ & 1 & \\
\hline Positive TST & $386(80.4)$ & 94 (19.6) & 3.5 & 2.4-4.99 \\
\hline No TST & $459(90.5)$ & $48(9.5)$ & 1.7 & $1.1-2.5$ \\
\hline
\end{tabular}

** The final multivariate logistics model includes the variables age, gender, TST in own history, TST history and country of birth.

In addition, two recently published incidence studies of Italian and Thai HCWs detected a higher risk of LTBI for specific occupational groups and units. One was a study with a homogeneous population of healthcare workers in a low-incidence area in Italy. Franchi and colleagues [37] found a low prevalence rate (6\%). Working in microbiology (OR 4.16, 95\% CI 1.27-13.6), dialysis/nephrology (OR 2.52, 95\% CI 1.36-4.65), gynaecology/obstetrics (OR 2.46, $95 \%$ CI 1.24-4.86) and age $>47$ years (OR 1.98, 95\% CI 1.14-3.46) were significant predictors for TB infection [30]. The second study investigated the association of workplace and the recent onset of LTBI in HCWs in a university hospital in Thailand. Working at in- and outpatient units and a history of tuberculosis exposure in the past year were significant predictors for tuberculin con- version [38]. However, these two studies were hampered by using the TST for LTBI diagnosis.

Another Italian study observed the prevalence among 115 HCWs tested simultaneously with both the TST and the IGRA. Overall, $53 \%$ of the individuals were TST-positive, $36.5 \%$ according to T-SPOT.TB and $25.2 \%$ according to QFT. An association between positives test results and an increased occupational risk of exposure to Mycobacterium tuberculosis was observed. Nurses and nurse assistants were more likely to be QFT-positive than physicians [39]. Demkow and colleagues found a higher risk of acquiring LTBI for TB lab workers (50\%) and TB ward staff (34\%) [40]. In our study the prevalence of LTBI in lab workers was lower (11.4\%), but we could not distinguish between TB lab and non-TB lab. 
In Germany all HCWs with a positive TST obtained chest $\mathrm{x}$-rays after contact with a TB-index case or otherwise every third year. After the introduction of the IGRA only HCWs with a positive IGRA obtain chest x-rays. Therefore, introducing IGRA for TB screening in Germany reduces the number of X-rays and the amount of preventive chemotherapy needed by a high percentage (from $24 \%$ to $9.9 \%$ ) without missing an active case of TB.

Despite our increasing knowledge, several key questions about latent TB infection remain unanswered. Particularly, it should be noted that TST and IGRA identify an adaptive immune response against $M$. tuberculosis, but not necessarily a latent infection. A positive result of the diagnostic tests is primarily a measure of an immunological response to stimulation by mycobacterial antigens that should not therefore be equated with the presence of live $M$. tuberculosis in the human host. It is also uncertain how long adaptive immune response towards mycobacterial antigens persist in the absence of live mycobacteria. For these reasons, according to the recently published TBNET consensus statement regarding latent TB the term "latent infection" would at best implicate "lasting tuberculosis immune response" [41].

\section{Limitation}

The main limitation of our study is the cross-sectional study design, as mentioned before. Because of this, changes over time cannot be taken into consideration. We could not discriminate between recent and old infections. Therefore, in spite of the big sample size of more than 2000 participants, no statement can be made regarding the risks at different kinds of workplaces and occupations in the healthcare sector.

\section{Conclusion}

In summary, our data corroborates the results of previous studies that the prevalence of LTBI in low-incidence countries depends on age. One important result of our study is that we found no positive IGRA results among trainees in the healthcare sector. Introducing IGRA for TB screening in Germany reduced the number of X-rays and the preventive chemotherapy by a high percentage (from 24\% to 9.9\%) while no active TB case was missed. Serial examinations are required in order to identify different kinds of occupations or workplaces especially at risk of LTBI.

\footnotetext{
Abbreviations

ABF: acid-fast bacillus; BCG: Bacillus Calmette-Guérin; Cl: confidence interval; HCW: healthcare worker; IFN: interferon; IGRA: interferon-gamma release assay; LTBI: latent tuberculosis infection; MTB: Mycobacterium tuberculosis; NTM: non-tubercular mycobacteria; OR: odds ratio; OSH: occupational safety and health; PPD: purified protein derivate; QFT-IT: QuantiFERON"TB Gold In-Tube; TB: tuberculosis; TST: tuberculin skin test; TU: tuberculin unit.
}

\section{Competing interests}

The authors declare that they have no competing interests.

\section{Authors' contributions}

$\mathrm{RD}$ and $\mathrm{MH}$ have made substantial contributions to the conception and design of the study and have been involved in revising the manuscript critically for important intellectual content. They have given final approval of the version to be published. AN has made substantial contributions to the conception and design, as well as to the analysis and interpretation of data. He has been involved in drafting the manuscript. He has given final approval of the version to be published. AS has made substantial contributions to the conception and design, the acquisition of data, as well as to the analysis and interpretation of data. She has been involved in drafting the manuscript. She has given final approval of the version to be published.

\section{Acknowledgements}

We wish to thank all the occupational physicians Ms G. Beckmann, Ms S. Nausester, Ms R. Wolfanger-Bennoit, Ms U. Hein-Rusinek, Ms A Stroop, Ms E. Wittkowski, Ms A. Patzak, Ms R. Cranen, Ms C Bauersfeld, Ms B. Mussotter, Ms V. Hartig, Ms B. Hudde and Ms U. Hermann for the medical examinations and data collection. We would especially like to thank all of the healthcare workers who participated.

\section{Author Details}

1 Institution for Statutory Accident Insurance and Prevention in the Health and Welfare Services, Department of Occupational Health Research, Pappelallee 35-37, 22089 Hamburg, Germany, 2University Medical Center HamburgEppendorf, Institute for Health Services Research in Dermatology and Nursing, Martinistraße 52, 20246 Hamburg, Germany and ${ }^{3}$ Department of Pulmonary Medicine, Hannover Medical School (MHH), Carl-Neuberg-Straße1, 30625 Hannover, Germany

\section{Received: 13 October 2009 Accepted: 30 April 2010}

Published: 30 April 2010

\section{References}

1. Seidler A, Nienhaus A, Diel R: Review of epidemiological studies on the occupational risk of tuberculosis in low-incidence areas. Respiration 2005, 72:431-446.

2. Diel R, Schneider S, Meywald-Walter K, Ruf CM, Rusch-Gerdes S, Niemann S: Epidemiology of tuberculosis in Hamburg, Germany: long-term population-based analysis applying classical and molecular epidemiological techniques. J Clin Microbiol 2002, 40:532-539.

3. Menzies D, Joshi R, Pai M: Risk of tuberculosis infection and disease associated with work in health care settings. Int J Tuberc Lung Dis 2007 11:593-605

4. MMWR: Guidelines for preventing the transmission of mycobacterium tuberculosis in health care settings. MMWR Morb Mortal Wkly Rep 2005 54:1-147.

5. Brodhun B, Altmann D, Haas W: Bericht zur Epidemiologie der Tuberkulose in Deutschland für 2007. Berlin 2008

6. Guidelines for Occupational Medical Examination - Prophylaxis in Occupational Medicine 4th edition. Stuttgart: Gentner Verlag; 2007

7. Diel R, Forßbohm M, Loytved G, Haas W, Hauer B, Maffei D, et al:: Recommendations for environmental contact tracing in tuberculosis. German Central Committee against Tuberculosis. Gesundheitswesen 2007, 69:488-503.

8. Menzies D: What does tuberculin reactivity after bacille CalmetteGuerin vaccination tell us? Clin Infect Dis 2000, 31(Suppl 3):S71-S74.

9. Nahid P, Pai M, Hopewell PC: Advances in the diagnosis and treatment of tuberculosis. Proc Am Thorac Soc 2006, 3:103-110.

10. Pai M, Kalantri S, Dheda K: New tools and emerging technologies for the diagnosis of tuberculosis: part II. Active tuberculosis and drug resistance. Expert Rev Mol Diagn 2006, 6:423-432.

11. National Institute for Health and Clinical Excellence: Clinical Guidelines 33. Tuberculosis: clinical diagnosis and management of tuberculosis, and measures for its prevention and control. London, UK 2007.

12. Menzies D, Pai M, Comstock G: Meta-analysis: new tests for the diagnosis of latent tuberculosis infection: areas of uncertainty and recommendations for research. Ann Intern Med 2007, 146:340-354. 
13. Andersen P, Munk ME, Pollock JM, Doherty TM: Specific immune-based diagnosis of tuberculosis. Lancet 2000, 356:1099-1104.

14. Mahairas GG, Sabo PJ, Hickey MJ, Singh DC, Stover CK: Molecular analysis of genetic differences between Mycobacterium bovis BCG and virulent M. bovis. J Bacteriol 1996, 178:1274-1282.

15. Nienhaus A, Loddenkemper R, Hauer B, Wolf N, Diel R: Latent tuberculosis infection in healthcare workers--evaluation of an Interferon-gamma release assay. Pneumologie 2007, 61:219-223.

16. Kobashi Y, Obase Y, Fukuda M, Yoshida K, Miyashita N, Fujii M, et al. Usefulness of QuantiFERON TB-2G, a diagnostic method for latent tuberculosis infection, in a contact investigation of health care workers. Intern Med 2007, 46:1543-1549.

17. Lee SS, Liu YC, Huang TS, Chen YS, Tsai HC, Wann SR, et al:: Comparison of the interferon- gamma release assay and the tuberculin skin test for contact investigation of tuberculosis in BCG-vaccinated health care workers. Scand J Infect Dis 2008, 40:373-380.

18. Vinton P, Mihrshahi S, Johnson P, Jenkin GA, Jolley D, Biggs BA: Comparison of QuantiFERON-TB Gold In-Tube Test and Tuberculin Skin Test for Identification of Latent Mycobacterium tuberculosis Infection in Healthcare Staff and Association Between Positive Test Results and Known Risk Factors for Infection. Infect Control Hosp Epidemiol 2009, 30:215-221.

19. Soborg $B$, Andersen AB, Larsen HK, Weldingh $K$, Andersen $P$, Kofoed $K$, et al: Detecting a low prevalence of latent tuberculosis among health care workers in Denmark detected by M. tuberculosis specific IFNgamma whole-blood test. Scand J Infect Dis 2007, 39:554-559.

20. Harada N, Nakajima Y, Higuchi K, Sekiya Y, Rothel J, Mori T: Screening for tuberculosis infection using whole-blood interferon-gamma and Mantoux testing among Japanese healthcare workers. Infect Control Hosp Epidemiol 2006, 27:442-448.

21. Barsegian V, Mathias KD, Wrighton-Smith P, Grosse-Wilde H, Lindemann M: Prevalence of latent tuberculosis infection in German radiologists. $J$ Hosp Infect 2008, 69:69-76.

22. Stebler A, Iseli P, Muhlemann K, Bodmer T: Whole-blood interferongamma release assay for baseline tuberculosis screening of healthcare workers at a swiss university hospital. Infect Control Hosp Epidemiol 2008, 29:681-683

23. Ringshausen FC, Schlosser S, Nienhaus A, Schablon A, SchultzeWerninghaus G, Rohde G: In-hospital contact investigation among health care workers after exposure to smear-negative tuberculosis. $J$ Occup Med Toxicol 2009, 4:11.

24. Schablon A, Beckmann G, Harling M, Diel R, Nienhaus A: Prevalence of Latent Tuberculosis Infection among Health Care Workers in a hospital for pulmonary diseases. J Occup Med Toxicol 2009, 4:1

25. Storla DG, Kristiansen I, Oftung F, Korsvold GE, Gaupset M, Gran G, et al:: Use of interferon gamma-based assay to diagnose tuberculosis infection in health care workers after short term exposure. BMC Infect Dis 2009, 9:60

26. Nienhaus A, Schablon A, Siano B, le Bacle C, Diel R: Evaluation of the Interferon-gamma Release Assay in Healthcare Workers. Int Arch Occup Enviro Health 2008, 81:295-300

27. Kralj N, Hofmann F, Michaelis M: Zur Methodik der Tuberkulosefrüherkennung bei arbeitsmedizinischen Vorsorgeuntersuchungen im Gesundheitsdienst. Arbeitsmed Sozialmed Umweltmed 1997, 32:50-54.

28. Lange C, Schaberg T, Diel R, Greinert U: Aktueller Stand der Tuberkulosediagnostik. Dtsch Med Wochenschr 2006, 131:341-347.

29. Pai M, O'Brien R: Serial testing for tuberculosis: can we make sense of $T$ cell assay conversions and reversions? PLoS Med 2007, 4:-e208.

30. Tripodi D, Brunet-Court, Nael V, Audrain M, Chailleux E, Germaud P, et al.: Evaluation of the tuberculin skin test and the interferon-gamma release assay for TB screening in French healthcare workers. $J$ Occup Med Toxicol 2009, 4:30.

31. Torres Costa J, Sa R, Cardoso MJ, Silva R, Ferreira J, Ribeiro C, et al: Tuberculosis screening in Portuguese healthcare workers using the tuberculin skin test and the interferon-gamma release assay. Eur Respir J 2009, 34:1423-1428.

32. Chee CB, Lim LK, Barkham TM, Koh DR, Lam SO, Shen L, et al:: Use of a T cell interferon-gamma release assay to evaluate tuberculosis risk in newly qualified physicians in Singapore healthcare institutions. Infect Control Hosp Epidemiol 2009, 30:870-875.
33. Nienhaus A, Schablon A, Loddenkemper R, Hauer B, Wolf N, Diel R: Prevalence of latent tuberculosis infection in healthcare workers in geriatric care. Pneumologie 2007, 61:613-616.

34. Alvarez-Leon EE, Espinosa-Vega E, Santana-Rodriguez E, Molina-Cabrillana $J M$, Perez-Arellano JL, Caminero JA, et al:: Screening for tuberculosis infection in spanish healthcare workers: Comparison of the QuantiFERON-TB gold in-tube test with the tuberculin skin test. Infect Control Hosp Epidemiol 2009, 30:876-883.

35. Ong A, Rudoy I, Gonzalez LC, Creasman J, Kawamura LM, Daley CL: Tuberculosis in healthcare workers: a molecular epidemiologic study in San Francisco. Infect Control Hosp Epidemiol 2006, 27:453-458.

36. De Vries G, Sebek MM, Lambregts-van Weezenbeek CS: Healthcare workers with tuberculosis infected during work. Eur Respir J 2006, 28:1216-1221

37. Franchi A, Diana O, Franco G: Job-related risk of latent tuberculosis infection in a homogeneous population of hospital workers in a low incidence area. Am J Ind Med 2009, 52:297-303.

38. Sawanyawisuth K, Chaiear N, Sawanyawisuth K, Limpawattana P, Bourpoern J, Reechaipichitkul W, et al: Can workplaces be predictors for recent onset latent tuberculosis in health care workers? J Occup Med Toxicol 2009, 4:20.

39. Girardi E, Angeletti C, Puro V, Sorrentino R, Magnavita N, Vincenti D, et al:: Estimating diagnostic accuracy of tests for latent tuberculosis infection without a gold standard among healthcare workers. Euro Surveill 2009, 14:.

40. Demkow U, Broniarek-Samson B, Filewska M, Lewandowska K, Maciejewski J, Zycinska K, et al:: Prevalence of latent tuberculosis infection in health care workers in Poland assessed by interferongamma whole blood and tuberculin skin tests. J Physiol Pharmacol 2008, 59(6):206-217

41. Mack U, Migliori GB, Sester M, Riedre HL, Ehlers S, Goletti D, et al: LTBI: latent tuberculosis infection or lasting immune responses to $M$. tuberculosis?- A TBNET consensus statement. Eur Respir J 2009, 33:956-973

\section{Pre-publication history}

The pre-publication history for this paper can be accessed here: http://www.biomedcentral.com/1471-2334/10/107/prepub

doi: 10.1186/1471-2334-10-107

Cite this article as: Schablon et al., Risk of latent TB infection in individuals employed in the healthcare sector in Germany: a multicentre prevalence study BMC Infectious Diseases 2010, 10:107

\section{Submit your next manuscript to BioMed Centra and take full advantage of:}

- Convenient online submission

- Thorough peer review

- No space constraints or color figure charges

- Immediate publication on acceptance

- Inclusion in PubMed, CAS, Scopus and Google Scholar

- Research which is freely available for redistribution 